\title{
IMAGING SPECTROSCOPY OF THE HUMAN OCULAR FUNDUS IN VIVO
}

\author{
Martin Hammer, ${ }^{\dagger}$ Dietrich Schweitzer ${ }^{\dagger}$ Lutz Leistritz $^{\dagger}$ Mateusz Scibor ${ }^{\dagger}$ Karl-Heinz \\ Donnerhacke, ${ }^{\dagger}$ and Jürgen Strobel ${ }^{\dagger}$ \\ ${ }^{\dagger}$ Friedrich Schiller University Jena, Bachstr. 18, Jena 07740, Germany; ${ }^{\ddagger}$ Carl Zeiss Jena GmbH, \\ Jena 07740, Germany \\ (Paper JBO-138 received Feb. 7, 1997; revised manuscript received June 30, 1997; accepted for publication July 29, 1997.)
}

\begin{abstract}
Spectroscopic measurement of light that is reflected from biological tissue in vivo is being investigated for various clinical applications. One special object of investigation using optical methods is the human ocular fundus. A fundus reflectometer that enables the simultaneous acquisition of up to 192 spectra arranged in a horizontal line across the fundus is described. The underlying optical principle of the device is the confocal imaging of an illuminated narrow, slitlike field at the fundus to the entrance slit of a spectrograph. This is imaged by the grating of the spectrograph onto a two-dimensional CCD chip that records the local distribution of ocular fundus reflectance spectra within a wavelength range of 400 up to $710 \mathrm{~nm}$ with a resolution better than $2 \mathrm{~nm}$ and a local resolution of $23 \mu \mathrm{m}$ in a field dimension of $1.5 \mathrm{~mm}$. The performance of the device was investigated, the effects of confocal and nonconfocal imaging are discussed, and some representative measurements are presented. (c) 1997 Society of Photo-Optical Instrumentation Engineers. [S1083-3668(97)01004-6]
\end{abstract}

Keywords reflectance spectroscopy; ocular fundus; instrumentation.

\section{INTRODUCTION}

While the ophthalmologist usually estimates the structure and the color of the ocular fundus, additional knowledge of the spectral distribution of the light that is reflected from the eye ground may be important in diagnostics as well as in laser therapy. Since the first spectrally resolved photometric measurements were made of light reflected from the human ocular fundus, in vivo reflectometry has been used to describe the physiological state of the fundus and pathologic processes. ${ }^{1-19}$ The extinction of melanin, ${ }^{7}$ xanthophyll, ${ }^{14,18}$ and hemoglobin ${ }^{2,14,18}$ inside the retina and choroid may be measured by spectrally resolved fundus reflectometry. In addition, the oxygen saturation of blood ${ }^{3,18,20-22}$ and the concentration and regeneration kinetics ${ }^{1,5,8,9,11,12,15}$ of photopigments can be determined and the effect of photocoagulation ${ }^{23-26}$ may be investigated.

Different types of fundus reflectometers and densitometers have been developed over the past 40 years. ${ }^{1,2,4-9,11-13,18,27}$ All these instruments, however, are restricted to measurement at a single location ${ }^{2,6,11,12,18,27}$ of the fundus or to a few wavelength bands that are determined by the transmittance of the interference filters used ${ }^{1,8,9}$ or laser wavelengths in a scanning laser ophthalmoscope. ${ }^{4,13}$ Furthermore, most of these devices illuminate a field at the fundus that is larger than the detection area, so that not only reflected light from

Address all correspondence to Martin Hammer. Tel \& Fax: 493641 633027; E-mail: hammer@bach.med.uni-jena.de the field under investigation contributes to the measurement, but also light that is scattered over a greater distance.

This paper describes a fundus reflectometer that is able to record the reflectance spectra of up to 192 sites arranged in a horizontal section across the fundus using imaging spectroscopy. ${ }^{28,29}$ This technique is based on the ability of the spectrograph to spectrally resolve and image its one-dimensional entrance slit onto a two-dimensional CCD matrix camera.

Imaging spectroscopy of the ocular fundus provides a unique opportunity to detect the local distribution of chromophores as well as differences in the scattering properties of the tissue within a single measurement. Errors due to head and eye movements in successive measurements at different locations are avoided. This is useful in oxymetric measurements in single retinal vessels, ${ }^{21,22}$ in the determination of the optical density of fundus pigments such as xanthophyll and melanin or metabolic deposits in exudates or drusen and their local distribution, and in the investigation of local differences in retinal scattering behavior as, for example, in nerve fiber bundles or edema. The performance of this apparatus was investigated and the influence of confocal and nonconfocal illumination is discussed. First, however, representative measurements are presented.

1083-3668/97/\$10.00 @ 1997 SPIE 


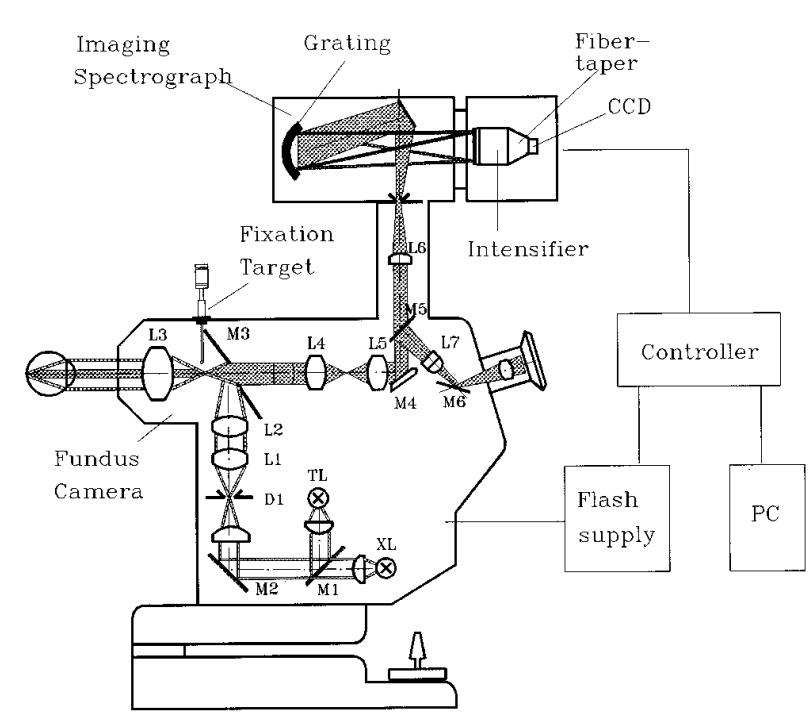

Fig. 1 Experimental setup of the imaging fundus reflectometer. See text for symbol explanations.

\section{EXPERIMENTAL SETUP}

\subsection{OPTICS}

The basic instrument used is the RCM 250 fundus camera (Carl Zeiss, Jena, Germany), which illuminates the fundus by (Figure 1) a tungsten halogen lamp (TL), mirrors M1, M2, and M3, and lenses L1, L2, and L3. Observation of the fundus is possible with a field angle of $45 \mathrm{deg}$ in the commonly known principle of aperture division by lenses L3, L4, L5, and L7, and mirrors M4, M5, and M6. In a plane conjugate to the retina, a slitlike field stop (D1) was added, which was inserted into the illuminating beam of the camera for the duration of the measurement only. Furthermore, mirrors M1 and M5 were removed and a field at the fundus with a size of $40 \times 1500 \mu \mathrm{m}^{2}$ was illuminated by a nonpolarized xenon arc flash lamp (XL). This fundus field was imaged confocally onto the entrance slit of the spectrograph CP 200 (Jobin Yvon, Longjumeau, France). The spectral range was 400 to $710 \mathrm{~nm}$ and the resolution was better than $2 \mathrm{~nm}$.

To achieve confocallity, a field-size adaptation was necessary and was provided by a system of two astigmatic lenses (L6). Furthermore, each point of the one-dimensional image of the illuminated retinal area in the entrance slit of the spectrograph was spectrally resolved and imaged onto one line of the front cathode of an intensified CCD camera (Princeton Instruments, Trenton, New Jersey). The light impinging on the S 20 cathode was enhanced by a multichannel plate intensifier and guided to the CCD matrix by a fiber taper, yielding a twodimensional image. The horizontal coordinate of this image represents the spectral dimension while the local resolution is drawn on the vertical coordinate. This optical concept, which is called imaging spectrometry, makes it possible to measure the local distribution of fundus reflectance spectra (referred to as stripes) with a single flash.

\subsection{ELECTRONICS AND DATA ACQUISITION}

The whole process of data acquisition was governed by the CCD detector controller. In a continuously running mode, the CCD matrix was cleared after a period of $100 \mathrm{~ms}$ of dark charge integration. When starting a new integration, a signal was sent to the flash lamp supply. After the adjustment of the patient's eye in front of the fundus camera, the operator pressed a button to initiate the measurement. This button signal was linked by an AND gate with the signal from the detector controller so that the start of the measurement procedure was synchronized with the start of the integration phase of the detector. The measurement started with the electrical insertion of the confocal field stop D1 and the removal of mirrors M1 and M5, followed by a xenon flash with a duration of at least $10 \mathrm{~ms}$. The whole procedure took some $10 \mathrm{~ms}$ so that the light flash that was reflected from the patient's ocular fundus was recorded by the CCD within its integration time of $100 \mathrm{~ms}$. Afterward the recorded image was digitized with a resolution of 14 bits and stored in a personal computer. To reduce noise, the charges of three neighboring stripes were integrated at the CCD before reading the chip (on-chip binning). This resulted in an image consisting of 64 stripes $(I[2] \ldots I[65])$ representing the spectra of 64 neighboring locations. In addition, one spectrum was simultaneously recorded at $3.4 \mathrm{deg}$ (about 1 $\mathrm{mm}$ in emmetropic eyes) to the illuminated area and was stored in stripe $I[1]$ for intraocular stray light correction purposes. The readout time of the whole image was $265 \mathrm{~ms}$.

\subsection{CALIBRATION, DATA PROCESSING, AND IN VIVO MEASUREMENT}

Before each measurement, the reflectance of a black wall about $1 \mathrm{~m}$ from the camera was recorded $\left(I_{b}\right)$. This wall reflected virtually no light into the measuring aperture of the camera so that the dark charge, internal stray light, and reflexes by the ophthalmoscope lens L3 were recorded as a background for the correction in all further measurements. All ocular fundus reflection data were related to that of a standard white reflection target with the Lambertian characteristic (Spectralon, Labsphere Inc., North Sutton, New Hampshire). The reflection $\left(I_{0}\right)$ of an artificial eye with this target in its focal plane was detected and set to unity. For the enhancement of the signal-to-noise ratio, the measurements of the background and the white standard target were averaged ten times.

Measurements in healthy persons and patients were performed with their agreement according to the guidelines of the Declaration of Helsinki and under the approval of a local ethics committee. The subject's pupil was dilated to a diameter of at least 
$6 \mathrm{~mm}$. The area of interest at the fundus was adjusted to a reticule in the ocular of the fundus camera that represented the location of the illuminated fundus field when the field stop was inserted during the measurement. For that reason, the subject was asked to look onto a fixation target, which was adjusted in a plane conjugate to the retina. Ten spectral images $(I)$ were recorded. If necessary, it was possible to correct the subject's fixation between the single measurements. The background $\left(I_{b}\right)$ was subtracted from all white standard $\left(I_{0}\right)$ and fundus reflection $(I)$ images. To compensate for intraocular stray light, the spectrum $I[1]$ of a nonilluminated location at the fundus was subtracted from the spectral images. The measurements from the ocular fundus were related to those from the artificial eye with the white fundus target. Each of the resulting ten images represented the local distribution of relative reflectance spectra at the chosen fundus site:

$$
R(\lambda)=\frac{I(\lambda)-I_{b}(\lambda)-I[1](\lambda)}{I_{0}(\lambda)-I_{b}(\lambda)-I_{0}[1](\lambda)} .
$$

However, in spite of the high local resolution of the method (see Sec. 3.3), even the smallest eye movements led to differences in the images. These were compensated by an alignment procedure in the following way: One of the ten images was chosen as the reference and all other images were shifted in the direction of the local resolution (vertically) until a maximum of correlation with the reference image was reached. All images that had a correlation coefficient beyond a predefined value $(0.988$ or greater) were averaged to improve the signal-tonoise ratio.

\section{PERFormanCE}

\subsection{RETINAL IRRADIANCE}

In order to estimate retinal irradiance during the examination, the energy of a single flash was measured in the pupillary plane by a RjP 735 EP detector (Laser Probe Inc., Utica, New York). This is a pyroelectric detector optimized for pulse lengths of up to $10 \mathrm{~ms}$. Since about $95 \%$ of the flash energy was emitted within the first $10 \mathrm{~ms}$, the energy values given by this detector may be considered to be realistic if they are triggered by the flash itself. Using the field stop described in Sec. 2.1, we measured an energy of $36.8 \pm 2 \mu \mathrm{J}$, corresponding to a retinal irradiance of $49 \mathrm{~mJ} / \mathrm{cm}^{2}$. This is about $25 \%$ of the maximum permissible exposure (MPE). ${ }^{30}$ A further reduction to $12.84 \pm 0.81 \mu \mathrm{J}$ was achieved by the use of a heat protection filter.

Although ANSI Z 136.1 deals with the exposure of the eye to laser light, it can be used in the following way: The illumination by a fundus camera with a $40 \times 1500-\mu \mathrm{m}^{2}$ field was treated as an extended source. For an exposure duration shorter than $10 \mathrm{~s}$, the MPE is independent of the wavelength between

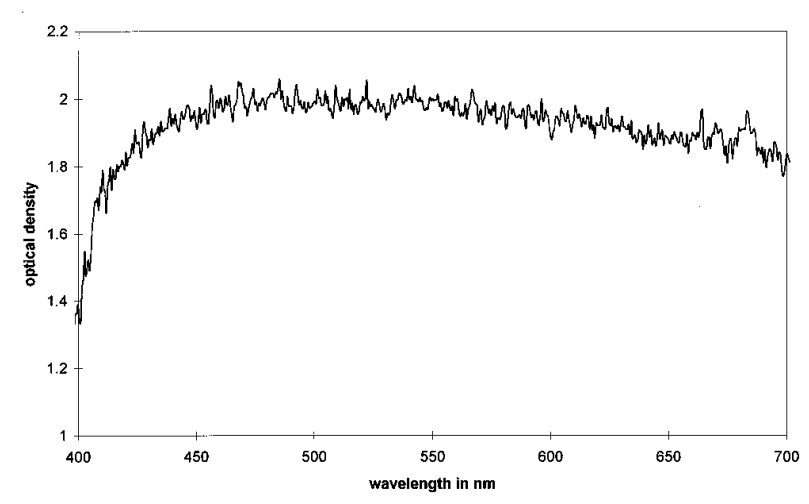

Fig. 2 Maximum resolvable optical density versus wavelength.

400 and $700 \mathrm{~nm}$. According to Delori, Parker, and Mainster $^{31}$ the spectral weighting function for the retinal damage susceptibility equals unity in the spectral range for exposures between $1 \mathrm{~ms}$ and $10 \mathrm{~s}$. In order to account for the effect of 10 exposures with a maximal repetition rate of one per second, the "long exposure" and the "short exposure" were calculated to be 32 and $2.5 \%$ of the MPE, respectively. ${ }^{30,31}$

\subsection{PHOTOMETRIC RESOLUTION}

First the maximum optical density that can be detected is considered. It is assumed to be the logarithm of half of the maximum signal-to-noise ratio:

$$
O D_{\max }=\log \frac{S N R_{\max }}{2}=\log \frac{\sqrt{n} I_{0}}{2\left(\sigma_{0}+\sigma_{b}\right)},
$$

where $\sigma_{0}$ is the standard deviation of $I_{0}, \sigma_{b}$ that of the background noise, and $n$ is the number of the averaged measurements. $O D_{\max }$ was determined to be about 2 for $n=10$ by the detection of 50 consecutive measurements of $I_{0}$ and $I_{b}$ (Figure 2). The wavelength dependence results from the spectral distribution of the radiation power of the lamp, the spectral transmission of the optics, and the spectral sensitivity of the detector. This, however, is not the optical density with respect to the radiation power entering the eye, but the density with respect to the power scattered by the white Lambertian reflectance target into the solid angle of the entrance aperture of the camera. It is about 1 percent of the incident power.

The smallest difference in the optical density that can be resolved in the case of a given density is determined by the measured signal $I_{O D}$ and the rms of its noise $\sigma_{O D}$ :

$$
\Delta O D(\lambda)=\log \frac{I_{O D}(\lambda)}{I_{O D}(\lambda)-2 \sigma_{O D}(\lambda)} .
$$

This was determined from the mean value and the standard deviation of 50 consecutive measurements using neutral density filters. The minimum detect- 


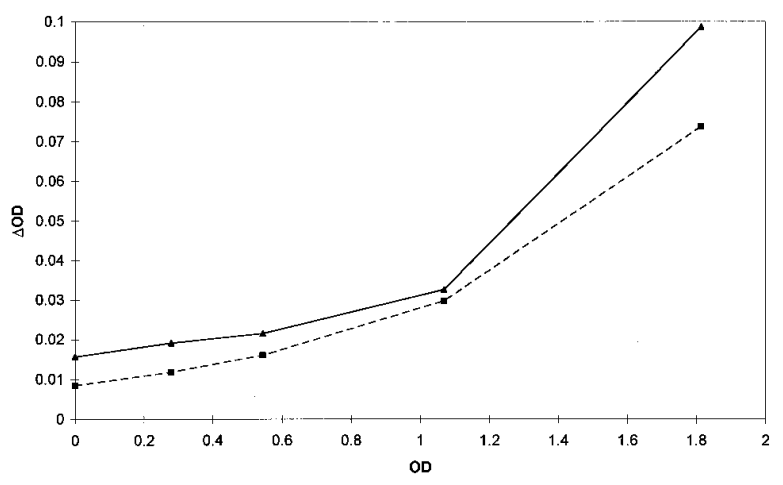

Fig. 3 Minimum measurable optical density difference versus optical density. Solid line, measurement; interrupted, theoretical value.

able optical density difference is shown in Figure 3 as a function of the optical density at a wavelength of $500 \mathrm{~nm}$ (solid line). The broken line represents a theoretical value assuming the noise to be the Poisson-distributed photon noise:

$$
\sigma_{O D_{t h}}=\sqrt{I_{O D}} .
$$

The main reason for the discrepancy of the experimental and the theoretical values is the limited stability of the flash lamp.

Photometric accuracy and linearity were checked using calibrated neutral density filters that were inserted into the illuminating light path. The nominal and measured extinction values and their differences at $500 \mathrm{~nm}$ are given in Table 1 . In any case, the differences between the nominal and the measured extinction are smaller than the minimal resolvable optical density differences. However, the measured density is always less than the nominal one, probably because of a reflex from the ophthalmoscope lens. Therefore this reflection was measured together with the background and subtracted from the signal in all further measurements.

\subsection{SPECTRAL AND LOCAL RESOLUTION}

As Abbe proposed, the light of the first diffraction order has to pass the aperture of an optical system for proper imaging of an object. ${ }^{32}$ Therefore, the

Table 1 Measured and nominal optical density of calibrated filters at $500 \mathrm{~nm}$.

\begin{tabular}{lll}
\hline$E_{\text {nominal }}$ & $E_{\text {measured }}$ & $\Delta E$ \\
\hline 0.3175 & 0.28 & 0.0375 \\
0.621 & 0.5969 & 0.0241 \\
0.9505 & 0.8994 & 0.0511 \\
1.4527 & 1.4058 & 0.0469 \\
1.9893 & 1.9289 & 0.0604 \\
2.5026 & 2.4348 & 0.0678 \\
\hline
\end{tabular}

angle of the first diffraction maximum has to be less than the aperture angle $\alpha$ of the optics, which is determined by the focal length $l$ of the eye and the entrance pupil of the ophthalmoscope:

$$
\sin \alpha=\frac{r}{l} .
$$

The diffraction angle $\phi_{k}$ of the $k^{\prime}$ th order of a slit with the width $b$ is given by ${ }^{32}$

$$
\sin \phi_{k}=\frac{2 k+1}{2} \frac{\lambda}{b} \text {. }
$$

According to Abbé, we set $\alpha=\phi_{1}$ and get

$$
b=\frac{3 \lambda l}{2 r}
$$

for the minimal width of a structure which should be resolved by our imaging system. We calculated $b=18.7 \mu \mathrm{m}$ for the fundus camera used, which possessed an aperture radius of $0.75 \mathrm{~mm}$ in the pupillary plane. This entrance pupil is a compromise between the theoretical resolution and image distortion by spherical aberration, which becomes significant at larger apertures. For the experimental determination of the lateral resolution of the imaging fundus spectrometer, we used the U.S. Air Force resolution target 1951 (Oriel Corporation, Stratford, Connecticut) in the focal plane of an artificial eye with a focal length of $20 \mathrm{~mm}$. All measurements at the target were corrected to the actual focal length of the human eye $(17 \mathrm{~mm})$.

One stripe at the CCD chip corresponds to a distance of $7.8 \mu \mathrm{m}$ at the ocular fundus. According to Rayleigh's criterion, a resolution of $20 \mu \mathrm{m}$ was measured. In order to improve the signal-to-noise ratio of our measurements, we chose a binning of each of three adjoining stripes. In this configuration, we achieved a resolution of $23.8 \mu \mathrm{m}$, which is in good agreement with the calculated distance at the fundus of $23.4 \mu \mathrm{m}$ and corresponds to three binned stripes at the CCD.

The spectral resolution of the spectrometer was checked using a mercury arc lamp. It was possible to resolve the mercury emission lines at 577 and 579 $\mathrm{nm}$ so that the spectral resolution was regarded to be better than $2 \mathrm{~nm}$.

Using a semi-confocal optical system (see Sec. 3.5) a depth resolution of $1.3 \mathrm{~mm}$ was achieved.

\subsection{APPLICATION OF THE POINT SPREAD FUNCTION FOR THE COMPENSATION OF IMAGE DISTORTIONS}

The quality of an optical system may be described by its point spread function (PSF). The PSF of the imaging fundus spectrometer was recorded by placing an illuminated vertical slit in the focal plane of an artificial eye that was narrowed to illuminate 


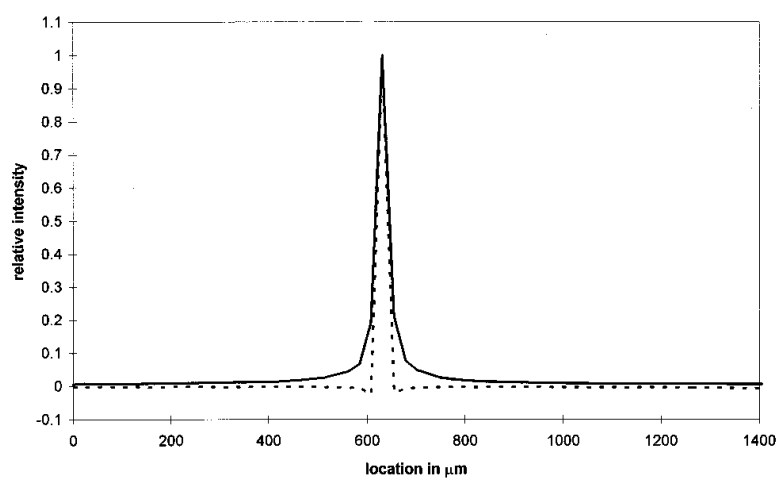

Fig. 4 Local intensity distribution of a spot as narrow as if it illuminated a single stripe at the CCD in the case of ideal geometrical optics (solid line) and the result of correction by multiplying the inverse PSF-matrix $A_{i, k}^{-1}$ (dotted line). Sections across the distribution of the spectra at $500 \mathrm{~nm}$.

only one stripe of the CCD camera. It is represented by the solid line in Figure 4 at $500 \mathrm{~nm}$.

In the case of a space-invariant system, the image $y$ of an object $x$ can be described by the following convolution integral:

$$
y=P S F \times x .
$$

In a more general sense, the image is a linear map of the object:

$$
y=A x
$$

In our case, the one-dimensional object can be described as a vector in $R^{N}$ where $N$ is the number of locations from which the spectra were recorded. Thus, the operator $A(\lambda)$ is an $N \times N$ matrix that can be obtained by measuring the PSF at all $N$ locations: Assuming $x^{(k)}(\lambda)$ to be the multiple of a $\delta$ function for all $k=1 \ldots N$, and measuring its image

$$
\begin{gathered}
y^{(k)}(\lambda)=\left[y_{1}^{(k)}(\lambda) \ldots y_{N}^{(k)}(\lambda)\right]^{T}, \\
A_{i, k}(\lambda) \sim y_{i}^{(k)}(\lambda)
\end{gathered}
$$

can be calculated. In order to correct the image $y(\lambda)$ of an arbitrary object $x(\lambda)$ for aberrations of the optics, the image has to be multiplied by the inverse matrix $A(\lambda)^{-1}$. This has to be done for all wavelengths separately. The dotted line in Figure 4 shows the PSF at $500 \mathrm{~nm}$ after the correction.

\subsection{COMPARISON OF CONFOCAL AND NONCONFOCAL IMAGING}

In confocal imaging a small spot is focused onto the object. Only the light that is reflected from this illuminated area is detected using a field stop in a plane conjugate to the object in front of the detector. The advantage of this technique is that stray light is removed. If the object is a scattering bulk material, the plane of interest can be observed by focusing the spot and the field stop on it. In our arrangement, the illuminating spot is produced by the slit-

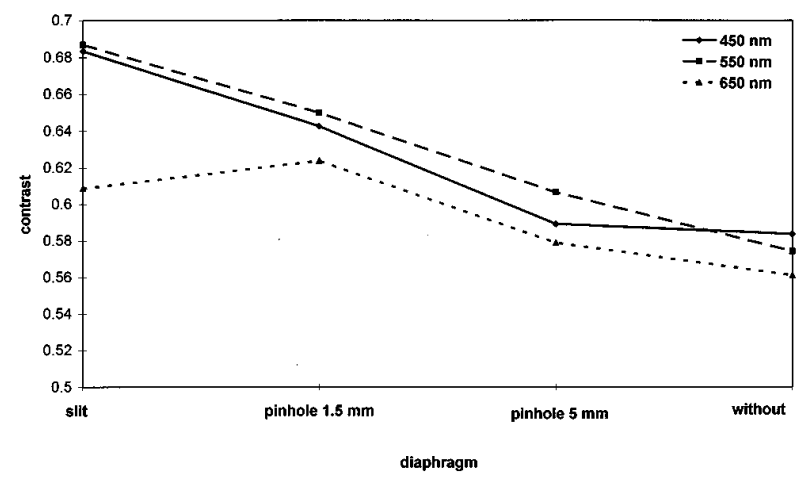

Fig. 5 Contrast of a hair in front of a white reflectance target versus illumination geometry.

shaped diaphragm (D1) in the fundus camera and the confocal field stop is the entrance slit of the spectrograph. Since both diaphragms are slits instead of pinholes in our apparatus, this setup will be referred to as semiconfocal. In the recent version of the device, D1 is focused onto the fundus of an emmetropic eye resulting in a strictly semi-confocal imaging only in this case. All fundus spectrometers described in the literature ${ }^{2,11,12,18}$ have different sizes of illuminated and observed fields so that their imaging is nonconfocal.

In order to investigate the effect of semiconfocal versus nonconfocal imaging, we removed the field diaphragm D1 and illuminated a large field at the fundus. The signal at the white standard reflection target was nine times higher than in the semiconfocal mode and the signal-to-noise ratio increased by a factor of 3 . This must be attributed to volume scattering in the target. However, the local resolution was even worse: 38 versus $23.8 \mu \mathrm{m}$ in the semiconfocal arrangement.

We also studied the contrast of a hair in front of a white reflection target using different D1 diaphragms. This contrast generally falls when the illuminated field becomes larger, as shown in Figure 5 for different wavelengths. The wavelength dependence of the contrast may be attributed to chromatic aberration of the optics. We found a pinhole with a diameter of $1.5 \mathrm{~mm}$ at the fundus to be a good compromise between the contrast and the signal-to-noise ratio. Therefore we measured the distribution of the spectra across two large blood vessels near the optic disk using the slit and the pinhole as field stop D1. The vessel profiles at 540 $\mathrm{nm}$ are depicted in Figure 6 and the reflection spectra recorded at the center of the artery are shown in Figure 7. The reflection measured in the case of nonconfocal illumination is much higher than in the semiconfocal case, indicating that the effect of volume scattering in the fundus tissue exceeds that in the white reference target. It indicates that most of the light detected with nonconfocal illumination is scattered from its surroundings into the field under investigation. This should be taken into account in 


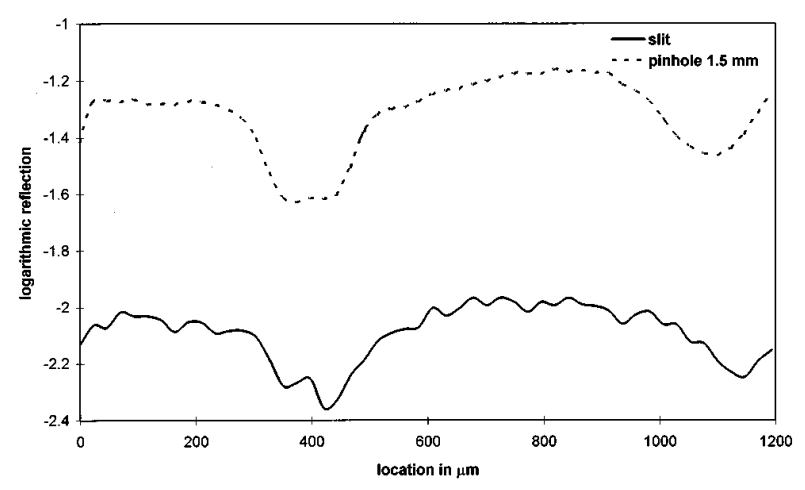

Fig. 6 Section across a fundus area containing two vessels at 540 $\mathrm{nm}$. Solid line, confocal illumination; dotted line, illumination by a pinhole with a diameter of $1.5 \mathrm{~mm}$.

the interpretation of the fundus reflectance spectra. Hammer et al. ${ }^{33}$ tried to fit a fundus reflectance model established by Delori and Pflisben ${ }^{2}$ to their measurements without success. On the other hand, the model fit the measurements given by Delori and Pflisben. ${ }^{2}$ The reason for this might be that the scattering term of the model according to the Kubelka-Munk theory is valid for the nonconfocal measurement but not for the confocal one.

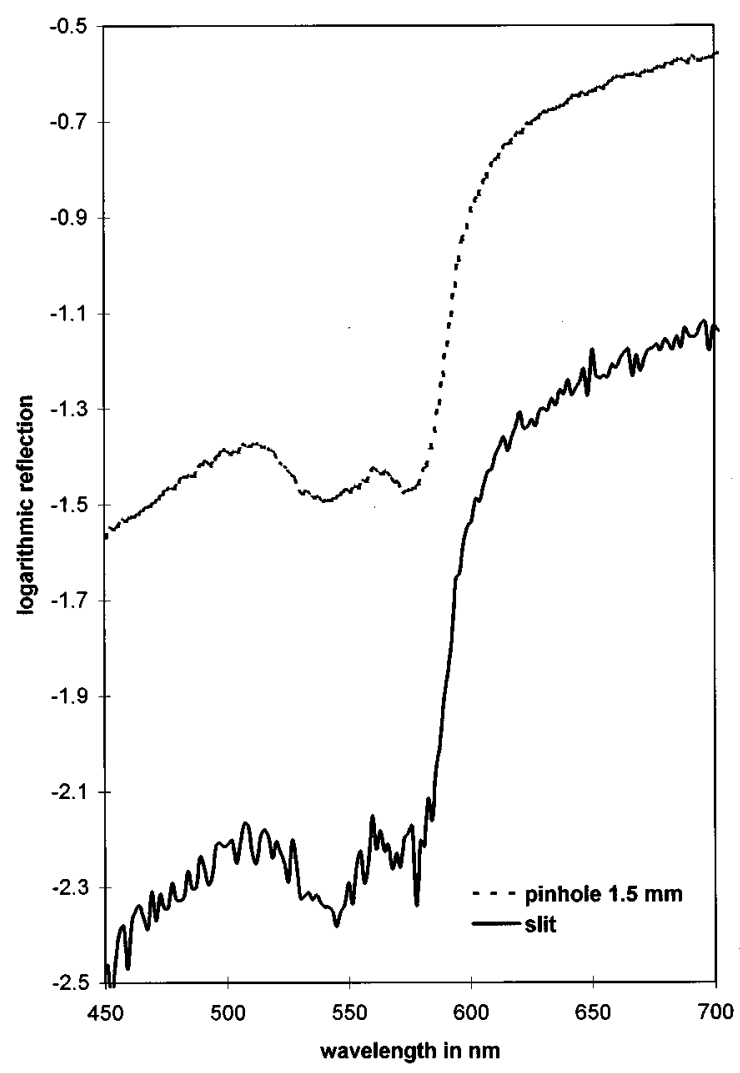

Fig. 7 Reflection of a retinal artery versus wavelength. Solid line, confocal illumination; dotted line, illumination by a pinhole with a diameter of $1.5 \mathrm{~mm}$.

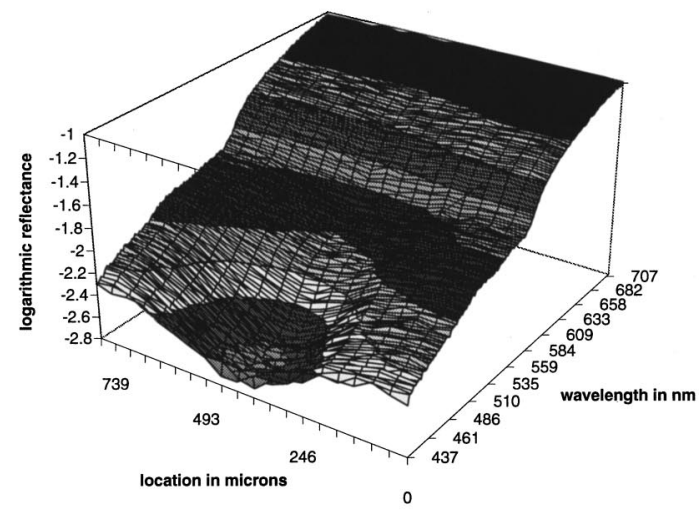

a
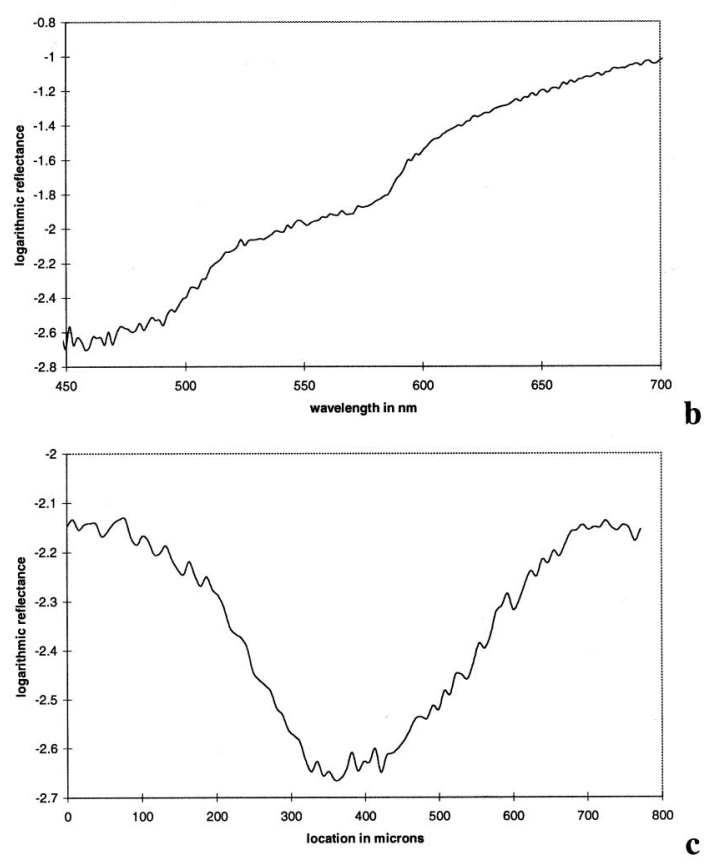

Fig. 8 Local distribution of the reflectance spectra from (a) a normal macula, (b) the spectrum of the central fovea, and (c) a local cross-section at $465 \mathrm{~nm}$. (Reprinted with permission from S. Karger AG, Basel.)

\section{REPRESENTATIVE MEASUREMENTS}

Some representative measurements are shown in Figures 8 through 11 . The fundus reflectance is depicted in a logarithmic scale as a function of wavelength and location. Figure 8 shows the reflectance spectra of a normal macula, the location of the highest visual acuity. The local distribution of the spectra (a) as well as the central spectrum (b) and the local distribution of the intensity of the reflected light at $465 \mathrm{~nm}$ (c) are presented. The reflection at wavelengths greater than $600 \mathrm{~nm}$ is determined by the absorption of melanin in the choroid and in the retinal pigment epithelium. Below $600 \mathrm{~nm}$, reflection strongly decreases due to hemoglobin absorption. The minima at 542 and $576 \mathrm{~nm}$ (see also Figure 7) correspond to the absorption maxima of oxygenated hemoglobin. Thus the spectral course of the 


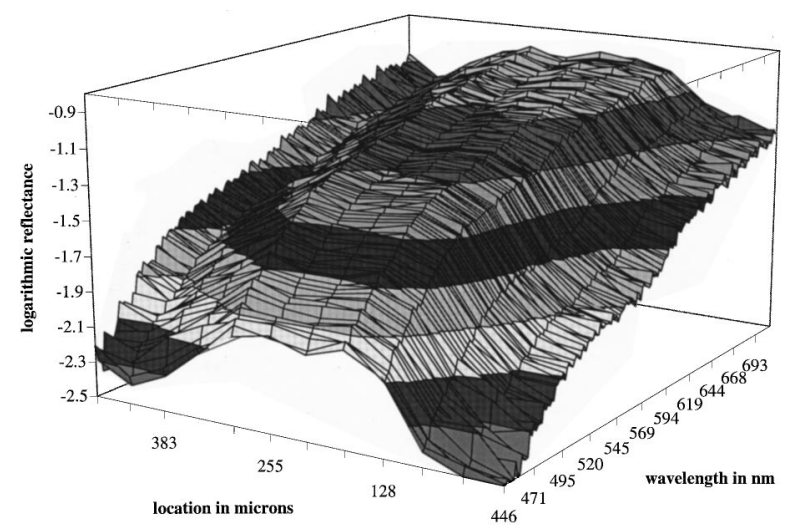

Fig. 9 Local distribution of the reflectance spectra from an exudate in a patient suffering from diabetic retinopathy.

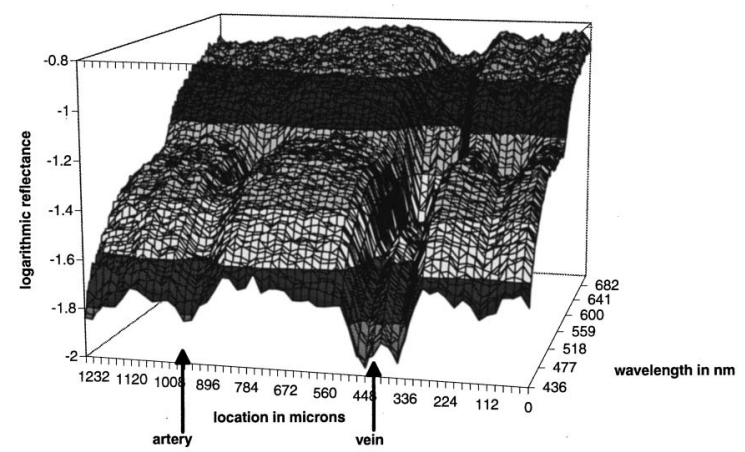

a
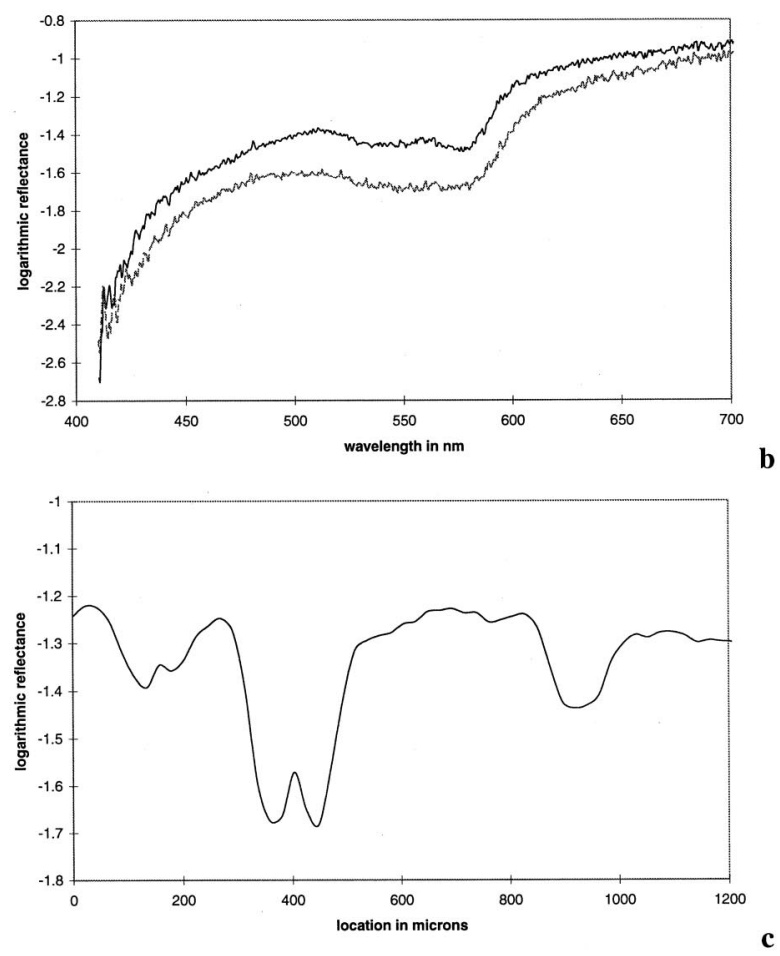

Fig. 10 Local distribution of the reflectance spectra from (a) blood vessels, (b) spectra of an artery (top) and a vein (bottom), and (c) a local cross section at $559 \mathrm{~nm}$.

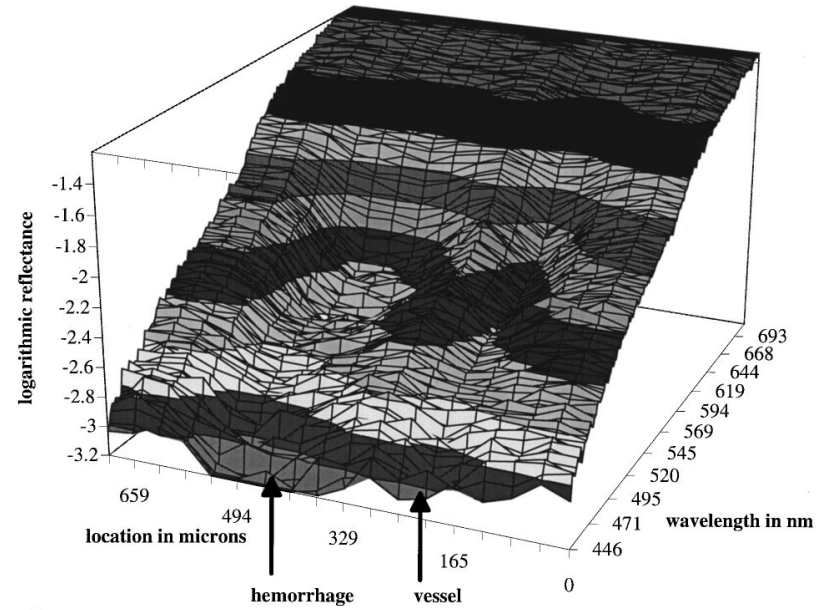

Fig. 11 Local distribution of the reflectance spectra from a thrombosis. The spectra of a hemorrhage can be seen to the left of the occluded vessel.

reflectance may be used to determine the oxygen saturation of blood. ${ }^{21,22}$ The reduction of reflectance in the fovea below $520 \mathrm{~nm}$ results from the absorption of light by xanthophyll. Furthermore, the spectral characteristics of the anterior ocular media contribute to the reflection spectra. The concentration of single pigments can be evaluated by fitting mathematical models of the fundus reflection to the measured spectra. $2,14,18,33,34$

In Figure 9, reflectance spectra of an exudate in a patient suffering from diabetic retinopathy are shown in the center of the local distribution. Here the spectral characteristics of the normal fundus shown at the edges are hidden by the nonabsorbing lipid that is deposited in the exudate. Figure 10 shows the local distribution of the reflectance spectra of blood vessels just below the optic disk (a), the spectra of an artery and a vein (b), and the vessel profile at $559 \mathrm{~nm}$. Measurements like these are suitable for calculation of the vessel diameter and the oxygen saturation of the blood inside the vessels. ${ }^{21,22}$ Finally, the spectra from a branch vein thrombosis are considered. In Figure 11, the "valley" at the right-hand side of the local spectral distribution is the reflectance of the occluded vessel while that at the left-hand side is due to a hemorrhage. Fitting a model that incorporates the absorption spectra of oxygenated and reduced hemoglobin to the measured reflection spectra by varying the model parameters, the local distribution of oxygen saturation of blood can be determined. In the thrombosis case shown, a reduction of hemoglobin oxygenation from the center of the hemorrhage to the occluded vein was found. ${ }^{34}$

\section{CONCLUSIONS}

The described method of imaging spectroscopy enables one to record the local distribution of spectra along a horizontal section across the human ocular fundus in vivo. According to initial measurements 
in patients, the method appears to be promising for the investigation of pathological mechanisms of ocular and systemic diseases, in diagnosis, and in the control as well as the optimization of therapy. A combination with optical coherence tomography ${ }^{35}$ to measure optical path lengths in vivo may be of special interest. Using this combination, the concentration of fundus pigments can be calculated from the reflectance spectra. However, further work has to be done, especially with respect to the effect of scattering and wave propagation in tissue on the reflectance spectra.

\section{Acknowledgments}

This work was supported by Grant 01 IN 202 A/4 from the Bundesministerium für Wissenschaft und Bildung of Germany.

\section{REFERENCES}

1. F. W. Campbell and W. A. Rushton, "Measurement of the scotopic pigment in the living human eye," J. Physiol. 130, 131-147 (1955).

2. F. C. Delori and K. P. Pflibsen, "Spectral reflectance of the human ocular fundus," Appl. Opt. 28, 1061-1077 (1989).

3. F. C. Delori, "Noninvasive technique for oximetry of blood in retinal vessels," Appl. Opt. 27, 1113-1125 (1988).

4. A. E. Elsner, S. A. Burns, G. W. Huges, and R. H. Webb, "Reflectometry with the scanning laser ophthalmoscope," Appl. Opt. 31, 3697-3710 (1992).

5. D. J. Faulkner and C. M. Kemp, "Human rhodopsin measurement using a T.V.-based imaging fundus reflectometer," Vision Res. 24, 221-231 (1984).

6. C. Hood and W. A. H. Rushton, "The Florida retinal densitometer," J. Physiol. 217, 213-229 (1971).

7. M. Hunold and P. Malessa, "Spectrometric determination of the melanin pigmentation of the human ocular fundus in vivo," Ophthal. Res. 6, 355-362 (1974).

8. P. E. Kilbride, L. P. Hutman, M. Fishman, and J. S. Read, "Foveal cone pigment density difference in the aging human eye," Vision Res. 26, 321-325 (1986).

9. P. E. Kilbride, J. S. Read, G. A. Fishman, and M. Fishman, "Determination of human cone pigment density difference spectra in spatially resolved regions in the fovea," Vision Res. 23, 1341-1350 (1983).

10. R. W. Knighton, S. G. Jacobson, and C. M. Kemp, "The spectral reflectance of the nerve fiber layer of the macaque retina," Invest. Ophthalmol. Vis. Sci. 30, 2393-2402 (1989).

11. D. van Norren and J. van de Kraats, "A continously recording densitometer," Vision Res. 21, 897-905 (1981).

12. D. van Norren and J. van de Kraats, "Retinal densitometer with the size of a fundus camera," Vision Res. 29, 369-374 (1989).

13. D. van Norren and J. van de Kraats, "Imaging retinal densitometry with a confocal scanning laser ophthalmoscope," Vision Res. 29, 1825-1830 (1989).

14. D. van Norren and L. F. Tiemeijer, "Spectral reflectance of the human eye," Vision Res. 26, 313-320 (1986).

15. W. A. H. Rushton, "The difference spectrum and the photosensitivity of rhodopsin in the living human eye," J. Physiol. 134, 11-29 (1956).

16. D. Schweitzer, S. Günther, M. Scibor, and M. Hammer, "Spectrometric investigations in ocular hypertension and early stages of primary open angle glaucoma and of low tension glaucoma-multisubstance analysis," Internat. Ophthalmol. 16, 251-257 (1992).

17. D. Schweitzer, S. Klein, S. Guenther, M. Hammer, and M. Scibor, "Early diagnosis of glaucoma by means of fundus reflectometry," New Trends Ophthalmol. 7, 241-247 (1992).

18. D. Schweitzer, E. Königsdörffer, G. Tröger, R. Augsten, S. Klein, and H. Roth, "Möglichkeiten und Grenzen der Fundusreflektometrie zum Nachweis von Veränderungen in einzelnen Schichten des Augenhintergrundes," Folia Ophthalmol. 15, 125-137 (1990).

19. D. Schweitzer, C. Schrödel, A. Jütte, F. Blaschke, and J. Opfermann, "Spektrometrie am Augenhintergrundneue diagnostische Möglichkeiten," Folia Ophthalmol. 10, 299-305 (1985).

20. J. P. de Kock, L. Tarassenko, C. J. Glynn, and A. R. Hill, "Reflectance pulse oximetry measurements from the retinal fundus," IEEE Trans. Biomed. Eng. 40, 817-823 (1993).

21. D. Schweitzer, L. Leistritz, M. Hammer, M. Scibor, U. Bartsch, and J. Strobel, "Calibration-free measurement of the oxygen saturation in retinal vessels of men," Proc. SPIE 2393, 210-218 (1995).

22. D. Schweitzer and M. Hammer, "Limits for the measurement of oxygen saturation by the imaging spectroscopy," Invest. Ophthalmol. 36/4, 105 (1995).

23. V.-P. Gabel, R. Birngruber, and B. Lorenz, "Klinische Relevanz einer Dosiereinrichtung für die Laserkoagulation," Klin. Mbl. Augenheilk. 188, 263-265 (1986).

24. A. W. Mainster, T. J. White, J. H. Tips, and P. W. Wilson, "Retinal temperature increase produced by intense light sources," JOSA 60, 264-270 (1970).

25. K. P. Pflibsen, F. C. Delori, O. Pomeranzeff, and M. M. Pankratov, "Fundus reflectometry for photocoagulation dosimetry," Appl. Opt. 28, 1084-1096 (1989).

26. O. Pomeranzeff, J. W. Guang-, M. Pankratov, and J. Schneider, "Time and location analysis of lesion formation in photocoagulation," Arch. Ophthalmol. 101, 954-957 (1983).

27. C. Riva, "New ocular fundus reflectometer," Appl. Opt. 11, 1845-1849 (1972).

28. D. Schweitzer and M. Hammer, "Anordnung zur spektrometrischen Untersuchung und deren Verwendung," Patentschrift DE4410690C1 (1994).

29. D. Schweitzer, M. Hammer, and M. Scibor, "Imaging spectroscopy in ophthalmology-principle and application in microcirculation and in investigation of pigments," Ophthalmic Res. 28, 37-44 (1996).

30. "American National Standard for the Safe Use of Lasers," ANSI Z 136.1-1993 1993 American National Standards Institute, New York.

31. F. C. Delori, J. S. Parker, and M. A. Mainster, "Light levels in fundus photography and fluorescein angiography," Vision Res. 20, 1099-1104 (1980).

32. H. Haferkorn, Optik, Chap. 6: "Optische instruments und systeme," p. 509, Verlag Harri Deutsch, Frankfurt (1981).

33. M. Hammer, D. Schweitzer, M. Scibor, L. Leistritz, and B. Hoffmann, "Comparison of different models for the radiative transfer to the human ocular fundus using a new technique for spectral reflectance measurement," in Proc. 4th International Meeting on Scanning Laser Ophthalmoscopy, Tomography and Microscopy, in press.

34. M. Hammer, D. Schweitzer, and M. Scibor, "Local resolved spectroscopy at the human ocular fundus in vivo-technique and clinical examples," Proc. SPIE 2632, 79-90 (1996).

35. D. Huang, E. A. Swanson, C. P. Lin, J. S. Schuman, W. G. Stinson, W. Chang, M. R. Hee, T. Flotte, C. A. Gregory, C. A. Pulafito, and J. G. Fujimoto, "Optical coherence tomography," Science 254, 1178-1181 (1991). 